\title{
Mechanical Energy and Propulsion in Ergometer Double Poling by Cross-country Skiers
}

\author{
JØRGEN DANIELSEN ${ }^{1}$, ØYVIND SANDBAKK ${ }^{1}$, HANS-CHRISTER HOLMBERG $^{2}$, and GERTJAN ETTEMA ${ }^{1}$ \\ ${ }^{1}$ Center for Elite Sports Research, Department of Neuroscience, Norwegian University of Science and Technology, \\ Trondheim, NORWAY; and ${ }^{2}$ Swedish Winter Sports Research Center, Department of Health Science, Mid Sweden \\ University, Östersund, SWEDEN
}

\begin{abstract}
DANIELSEN, J., Ø. SANDBAKK, H.-C. HOLMBERG, and G. ETTEMA. Mechanical Energy and Propulsion in Ergometer Double Poling by Cross-country Skiers. Med. Sci. Sports Exerc., Vol. 47, No. 12, pp. 2586-2594, 2015. Purpose: This study aims to investigate fluctuations in total mechanical energy of the body $\left(E_{\mathrm{body}}\right)$ in relation to external ergometer work $\left(W_{\text {erg }}\right)$ during the poling and recovery phases of simulated double-poling cross-country skiing. Methods: Nine male cross-country skiers (mean \pm SD age, $24 \pm 5$ yr; mean \pm SD body mass, $81.7 \pm 6.5 \mathrm{~kg}$ ) performed 4-min submaximal tests at low-intensity, moderate-intensity, and high-intensity levels and a 3-min all-out test on a ski ergometer. Motion capture analysis and load cell recordings were used to measure body kinematics and dynamics. From these, $W_{\text {erg }}, E_{\text {body }}$ (sum of the translational, rotational, and gravitational potential energies of all segments), and their time differentials (power $P$ ) were calculated. $P_{\text {tot }}$ - the rate of energy absorption or generation by muscles-tendons - was defined as the sum of $P_{\text {body }}$ and $P_{\text {erg. }}$. Results: $E_{\text {body }}$ showed large fluctuations over the movement cycle, decreasing during poling and increasing during the recovery phase. The fluctuation in $P_{\text {body }}$ was almost perfectly out of phase with $P_{\text {erg. }}$. Some muscle-tendon energy absorption was observed at the onset of poling. For the rest of poling and throughout the recovery phase, muscles-tendons generated energy to do $W_{\text {erg }}$ and to increase $E_{\text {body }}$. Approximately $50 \%$ of cycle $P_{\text {tot }}$ occurred during recovery for all intensity levels. Conclusions: In double poling, the extensive contribution of the lower extremities and trunk to whole-body muscle-tendon work during recovery facilitates a "direct" transfer of $E_{\text {body }}$ to $W_{\text {erg }}$ during the poling phase. This observation reveals that double poling involves a unique movement pattern different from most other forms of legged terrestrial locomotion, which are characterized primarily by inverted pendulum or spring-mass types of movement. Key Words: CROSS-COUNTRY SKIING, DYNAMICS, MECHANICAL WORK, BIOMECHANICS, LOCOMOTION
\end{abstract}

$\checkmark$ ait patterns involved in terrestrial locomotion of bipedal and quadrupedal mammals can be described in terms of fluctuations in the mechanical energy of the body or the body's center of mass (CoM). For decades, these fluctuations, in connection with many different modes of locomotion including walking, running, and hopping $(1,3,5,7-9,24)$, have been extensively characterized. Total mechanical energy of the body $\left(E_{\text {body }}\right)$ at any instant in time is the sum of the gravitational potential energy $\left(E_{\mathrm{pot}}\right)$ and translational and rotational kinetic energy $\left(E_{\text {kin }}\right)$ of all body segments.

Two basic forms of energy-saving mechanisms have been widely characterized: the inverted pendulum-like behavior employed during walking and the spring-mass behavior

Address for correspondence: Gertjan Ettema, Ph.D., Department of Neuroscience, Norwegian University of Science and Technology, Trondheim 7489, Norway; E-mail: gertjan.ettema@ntnu.no.

Submitted for publication February 2015.

Accepted for publication June 2015.

0195-9131/15/4712-2586/0

MEDICINE \& SCIENCE IN SPORTS \& EXERCISE ${ }_{\circledast}$

Copyright (C) 2015 by the American College of Sports Medicine

DOI: 10.1249/MSS.0000000000000723 used during running (e.g., Refs. $(3,7))$. Both mechanisms minimize the amount of active muscle-tendon work over the movement cycle but in distinctly different ways. The inverted pendulum mechanism allows for direct exchange between $E_{\text {kin }}$ and $E_{\text {pot }}$, the levels of which fluctuate out of phase, thereby minimizing overall fluctuations in $E_{\text {body }}$. Thus, the need to generate additional mechanical energy through, for example, skeletal muscle contraction is minimized. In the case of spring-mass behavior, however, $E_{\text {kin }}$ and $E_{\mathrm{pot}}$ simultaneously decrease after foot-ground contact, both reaching their lowest levels during midstance; as a result, $E_{\text {body }}$ fluctuates substantially (e.g., Refs. $(2,4)$ ). The reduction in $E_{\text {body }}$ does not necessarily indicate dissipation in the form of heat because the series elastic components in muscle-tendon systems can absorb energy during braking for reutilization during push-off through stretch-shortening contractions (e.g., Refs. $(7,11,12,27))$.

Over the years, humans have invented passive tools for more economical transport, such as wheels in cycling and skis and poles in cross-country skiing (XC skiing). XC skiing consists of two main styles - classical and skating, each with several subtechniques used on different inclines. Some of these passive tools minimize fluctuations in $E_{\text {body }}$. The skis in XC skiing allow for gliding over a major part of the 
movement cycle, thus avoiding some of the ground collisions and related $E_{\text {body }}$ reduction that take place in walking and running. Furthermore, in many $\mathrm{XC}$ skiing techniques, the poles allow for distribution of propulsive forces between the upper body and the lower body and over a larger portion of the cycle. These aspects reduce the metabolic cost of XC skiing compared to walking and running (20).

One of the most commonly employed classical techniques of XC skiing is double poling (DP), which involves synchronous and symmetrical poling movements and is used mainly on flat or slightly uphill terrain. In contrast to all other XC skiing techniques, during DP, propulsive forces are generated solely through the poles in contact with the ground, with the skis gliding continuously forward. Although the upper body plays a critical role in DP, the lower extremities are responsible for about $50 \%$ of the metabolic work (25) and play an augmented role for optimal generation of poling force at increasing intensities $(15,16)$.

The primary function of this active legwork is to increase the vertical oscillation of the CoM by extending the lower extremities in one part of the movement cycle before flexing them later in the cycle $(15,16,18)$. If this is the case, $E_{\text {body }}$ should increase during the recovery phase (no pole-ground contact) and decrease during the poling phase (pole-ground contact), suggesting that $E_{\text {body }}$ might be utilized to increase the external force applied by the poles to the ground. Hence, in contrast to running and diagonal skiing, during DP, propulsion is generated mainly as $E_{\text {body }}$ decreases, as shown recently by Pellegrini et al. (22). As a consequence, $E_{\text {body }}$ may be transferred directly into doing external mechanical work (29), rather than via storage and reutilization as elastic energy. The fluctuations in $E_{\text {body }}$ during DP, indicating direct transfer of $E_{\text {body }}$ to external work, are not in complete agreement with the proposal that use of passive tools minimizes the fluctuations in $E_{\text {body }}$. Such minimization is normally related to classical energy-saving mechanisms such as the inverted pendulum or the spring-mass behavior. In contrast, Pellegrini et al. (22) concluded that DP mimics a pendular gait because trunk movements during poling and recovery cause out-of-phase fluctuations in kinetic energy and potential energy. However, in DP, the mechanism for a pendular gait seems absent, making this interpretation uncertain. To our knowledge, no previous studies have included instantaneous measurements of doing external work, which would improve the interpretation of energy fluctuations during DP.

To gain further insight into the mechanics underlying DP $\mathrm{XC}$ skiing, we examined fluctuations in mechanical energy during ergometer DP, where external ergometer work $\left(W_{\text {erg }}\right)$ can be measured directly and instantaneously. Because ergometer poling differs from DP on roller skis or snow, $W_{\text {erg }}$ mimics work against the friction of snow and the increases in kinetic energy in the direction of movement, thereby allowing fluctuations in body mechanical energy and external mechanical work to be assessed separately. Our hypothesis was that fluctuations in $E_{\text {body }}$ are out of phase with generation of external work (i.e., fluctuations in ergometer energy), in contrast to other forms of locomotion such as walking, running, or diagonal skiing. Thus, "direct" transfer of $E_{\text {body }}$ to external mechanical work would be the major factor contributing to propulsion. The effect of intensity (i.e., increased ergometer power output) on mechanical energy fluctuations was investigated by comparing submaximal and maximal DP. We hypothesized that the vertical oscillation of the $\mathrm{CoM}$ and related fluctuations in mechanical energy would increase with increasing exercise intensity.

\section{METHODS}

Participants. Nine male Norwegian national-level cross-country skiers (mean $\pm \mathrm{SD}$ : age, $24 \pm 5 \mathrm{yr}$; body mass, $81.7 \pm 6.5 \mathrm{~kg}$; height, $1.86 \pm 0.06 \mathrm{~m} ; \dot{\mathrm{VO}}_{2 \max }$ running, $5.45 \pm 0.64 \mathrm{~L} \cdot \mathrm{min}^{-1}$ ) participated voluntarily in this study after providing a written informed consent. The participants were first verbally informed in full about the nature of the study and their right to withdraw at any point without giving any reason. The study's experimental protocol was approved by the Regional Committee for Medical and Health Research Ethics in Central Norway.

Experimental protocol. After completing a 15-min low-intensity warm-up by running on a treadmill and DP, the participants performed three 4-min submaximal trials at low-intensity (LOW), moderate-intensity (MOD), and highintensity (HIGH) levels, with 1-min to 2-min intervals of rest. Thereafter, an active recovery period of $5 \mathrm{~min}$ was allowed before the participants completed a single 3-min closed-end performance test (MAX). Kinetic and kinematic measurements were collected for approximately $30 \mathrm{~s}$ after steady-state external power production was achieved during each trial. Physiological variables were also assessed continuously during each trial.

DP was performed on a Concept2 SkiErg (Concept2 Inc., Morrisville, VT) mounted to the wall. The aeroresistance of the ergometer was set at the lowest level, thereby reducing poling times to resemble those used during DP on snow as closely as possible (14). A load cell capturing poling force and reflective markers indicating poling displacement were utilized to measure instantaneous production of mechanical power (for details, see "Kinetic measurements"). To ensure that the participants maintained the same position in front of the ergometer, a steel plate was placed on the floor in front of the foot at a distance from the ergometer that simulated DP movements on snow (14).

With the use of Borg's 6-20 scale for RPE, submaximal trials were individually matched at the same subjective level of exercise intensity (i.e., 10, 13, and 16), which corresponds to intensities 1-3 in the Norwegian Olympic Committee intensity system (26). Thus, each participant generated external power in relationship to his own level of performance. All of the athletes had performed extensive endurance training for at least $6 \mathrm{yr}$ and were considered experienced in subjective control of intensity. MAX was performed at maximal 
effort, although the participants spent the initial $\sim 20 \mathrm{~s}$ to attain a power production they deemed sustainable for $3 \mathrm{~min}$. Respiratory variables and heart rate were measured continuously, and blood samples were collected immediately after each trial to measure lactate as an objective indicator of intensity. The participants performed all trials at their own freely chosen cycle rate. The integrated SkiErg performance monitor (PM4) displayed the mean DP ergometer power, allowing each subject to monitor and maintain power production as constant as possible throughout the submaximal trials, as instructed. The participants also received standardized encouragement during MAX.

Physiological measurements. Respiratory variables were continuously measured by open-circuit indirect calorimetry using an Oxycon Pro apparatus (Jaeger GmbH, Hoechberg, Germany). At the beginning of each test day, $\mathrm{O}_{2}$ and $\mathrm{CO}_{2}$ gas analyzers were calibrated against a known mixture of gases (mean $\pm \mathrm{SD}, 16.00 \% \pm 0.04 \% \mathrm{O}_{2}$ and $5.00 \% \pm 0.1 \% \mathrm{CO}_{2}$; Riessner-Gase GmbH \& Co, Lichtenfels, Germany), and the expiratory flow meter was calibrated with a 3-L volume syringe (Hans Rudolph Inc., Kansas City, MO). Blood lactate values were obtained from a $20-\mu \mathrm{L}$ blood sample collected from the fingertip and analyzed using a Biosen $\mathrm{C}_{-}$line Sport lactate analyzer (EKF-Diagnostic GmbH, Barleben, Germany). Heart rate was continuously recorded using a Suunto t6c heart rate monitor (Suunto Oy, Vantaa, Finland) and synchronized with the respiratory measurement system.

Kinetic measurements. For measurement of poling force $\left(F_{\text {poling }}\right)$, a Futek Miniature Tension and Compression Load Cell (Futek LCM200; capacity, 250 lb; nonlinearity, \pm $0.5 \%$; hysteresis, $\pm 0.5 \%$; weight, $17 \mathrm{~g}$; Futek Inc., Irvine, CA) was mounted on the ergometer in series with the drive cord inside the casing using a Rod End Bearing (GOD00730; Futek Inc.). The load cell was calibrated against a range of forces of known magnitude employing calibrated weights. Force data were sampled at $500 \mathrm{~Hz}$ and low-pass-filtered (cutoff frequency of $15 \mathrm{~Hz}$, eighth order, zero-lag Butterworth).

The two approaches most commonly employed to estimate fluctuations in the mechanical energy of the body and/ or the CoM are the CoM approach, which utilizes force platforms (e.g., Ref. (6)), and the sum of segmental energies, which utilizes motion capture (e.g., Ref. (28)). Because DP involves considerable trunk extension and flexion (e.g., Refs. $(16,25))$ and thereby possibly substantial rotational kinetic energy $\left(E_{\text {rot }}\right)$, which may influence estimation of $E_{\text {body }}$, we adopted the second approach here. Seven infrared Oqus cameras were placed around the subjects to capture the threedimensional positions of passive reflective markers at a sampling frequency of $100 \mathrm{~Hz}$. Four markers were fixed on the ergometer to measure poling movement: two on the right and left handles and two on the right and left points where the ropes entered the ergometer.

Bilateral movement symmetry was assumed, analysis was restricted to the left side of the body, and all data were analyzed in the sagittal plane. Seven spherical reflective markers were placed on the left side of the body at anatomical landmarks using double-sided tape (3M). These landmarks included the shoe at the distal end of the fifth metatarsal of the foot, the lateral malleolus (ankle), the lateral epicondyle (knee), the greater trochanter (hip), the lateral end of the acromion process (shoulder), the lateral epicondyle of the humerus (elbow), and the styloid process of the ulna (wrist). Kinematic data were low-pass-filtered (cutoff frequency $25 \mathrm{~Hz}$, eighth order, zero-lag Butterworth). Kinematics and dynamics were recorded from the very start of the trials, and 20 DP cycles characterized by steady-state production of power were analyzed. All force and movement data were recorded and synchronized using the Qualisys Track Manager software (Qualisys AB, Gothenburg, Sweden). Offline data processing was performed in MATLAB 8.1.0. (R2013a; Mathworks Inc., Natick, MA).

Data analysis. The body was approximated as a system of linked rigid segments connected by frictionless revolute joints. The sagittal plane limb segments were defined as foot, leg, thigh, trunk (including head), upper arm, and forearm. The lengths of the segments were determined based on kinematic data and averaged over the entire period of analysis. Segmental masses, moments of inertia, and CoM were calculated according to de Leva (10) using individual body mass and segment lengths. Linear and angular velocities, accelerations of limb segments, and the velocity of poling handles relative to the ergometer were calculated by numerical differentiation of position data with respect to time (23). $W_{\text {erg }}$ was calculated from poling force and handle displacement. $E_{\text {body }}$ was calculated as the sum of translational kinetic energy in the horizontal and vertical directions $\left(E_{\text {kin-t }}\right)$ and the potential energy $\left(E_{\text {pot }}\right)$ and rotational kinetic energy $\left(E_{\text {kin-r }}\right)$ of all segments $i$ :

$$
E_{\mathrm{body}}=\sum\left(E_{\mathrm{kin}-\mathrm{t} i}+E_{\mathrm{pot} i}+E_{\mathrm{kin}-\mathrm{i} i}\right)=\sum\left(1 / 2 m_{i} v_{i}^{2}+m_{i} g h_{i}+1 / 2 I_{i} \omega_{i}^{2}\right)
$$

where $m$ is segment mass, $v$ is segment velocity in the sagittal plane, $I$ is segment moment of inertia, $\omega$ is segment angular velocity, $g$ is the gravitational acceleration constant $\left(9.81 \mathrm{~m} \cdot \mathrm{s}^{-2}\right), h$ is the height of segment CoM, and $i$ is individual segment. All energies were then differentiated with respect to time to yield their rate of change (power $P$ ). $P_{\text {tot }}$,

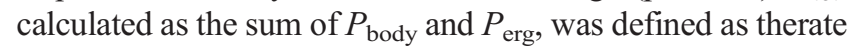
of mechanical energy that is generated or absorbed by deformation of body components, presumably by muscle-tendon systems or friction (for further consideration, see "Discussion").

One DP cycle was defined from the start of displacement (i.e., the highest position of poling handles relative to the following highest position). The poling phase was defined as the pull-down phase (i.e., the from highest position to the lowest position of the handles). The recovery phase was defined as the period of the opposite upward movement of the handles. Poling time (PT) was defined as the duration of the poling phase, cycle time (CT) was defined as the time of an entire poling + recovery movement, relative PT was defined as the percentage of CT, and cycle rate (CR) was defined as the number of poling cycles per second. 
Statistical analysis. All data were checked for normality using Shapiro-Wilk test and are presented as mean \pm SD. Repeated-measures ANOVA was used to determine whether there were any significant changes in variables as intensity and ergometer power output increased, and Bonferroni post hoc test was then applied to identify at which intensity any difference would be located. Statistical significance was set at $\alpha<0.05$, and all statistical tests were performed using SPSS version 21.0 (SPSS Inc., Chicago, IL).

\section{RESULTS}

Basic physiological and biomechanical characteristics. The cycle characteristics and physiological responses for all trials are presented in Table 1. PT decreased whereas $\mathrm{CR}$ increased progressively from LOW to MAX (all $P<0.001$ ). Relative PT at submaximal intensities did not differ but was higher at MAX $(P<0.001)$. RPE was close to the designated value at all intensities; external power, $\dot{\mathrm{V}} \mathrm{O}_{2}$, $\mathrm{HR}$, and blood lactate values increased steadily with intensity $(P<0.01)$.

Mechanical energy fluctuations. The behavior and timing of changes in $E_{\mathrm{kin}}, E_{\mathrm{pot}}$, and $E_{\mathrm{body}}$, together with $F_{\text {poling, }}$, are shown for all intensities in Figure 1, and the rates of change in the corresponding energies are shown in Figure 2. Early in the DP cycle $(\sim 10 \%), E_{\text {body }}$ remained almost constant, indicating direct energy exchange between $E_{\text {kin }}$ (increasing) and $E_{\text {pot }}$ (decreasing). $F_{\text {poling increased rapidly after about }}$ $\sim 10 \%$ of the cycle and coincided with a decrease in $E_{\text {pot }}$. Correspondingly, $E_{\text {kin }}$ reached the maximal value during this rapid increase in $F_{\text {poling. }}$. During the major part of the poling phase, $E_{\text {pot }}$ decreased, reaching its minimum value before the end of the poling phase (Figs. 1 and 2). Thus, during the first part of the poling phase, the CoM accelerated downwards; in the middle period, it decelerated downwards ( $E_{\text {kin }}$ and $E_{\text {pot }}$ decrease); and in the last portion, it accelerated upwards before propulsion was completed. The amount of energy involved in this action increased with exercise intensity and ergometer power output (Table 2). The amplitudes for fluctuations in $E_{\text {pot }}$ were about two to three times greater than those in $E_{\text {kin }}$.

Figure 1 illustrates fluctuations in mechanical energy relative to the start of a poling cycle. Thus, this figure does not indicate the vertical position of CoM. Maximal and minimal CoM positions are presented in Table 2. These maximal heights were the same for all submaximal intensities but higher for MAX $(P<0.05)$. Nonetheless, the vertical movement of the CoM, and thus energy flux, increased with intensity $(P<0.05)$ mainly because the minimal height during the poling phase decreased (Table 2).

During recovery, $E_{\text {pot }}$ increased considerably as the CoM rose. However, this increase started before the onset of recovery (i.e., when the ergometer handles were at their lowest position) and was finished before the end of recovery. In Figure 2, this is shown by the time point of the change in the sign of $P_{\text {pot }}$ relative to the poling-recovery transition. This pattern was independent of intensity. $E_{\text {kin }}$ changed very little during the transition between poling and recovery at submaximal intensities ( $P_{\text {kin }}$ close to zero; Fig. 2$)$. However, at MAX, $P_{\text {kin }}$ also exhibited a positive peak during this transition in the beginning of the recovery phase (Fig. $2 ; E_{\text {kin }}$ increasing, Fig. 1). At the end of recovery, $E_{\text {kin }}$ also increased simultaneously with a decrease in $E_{\text {pot }}$.

Muscle-tendon and external mechanical power and work. Figure 3 shows external ergometer power $\left(P_{\text {erg }}\right)$, total mechanical body power $\left(P_{\text {body }}\right)$, and their sum $\left(P_{\text {tot }}\right)$ (i.e., the rate of mechanical energy generation or absorption by muscles-tendons). For the majority of the poling phase, $P_{\text {body }}$ is negative. However, during the initial portion of the poling phase (first $\sim 10 \%$ ), $P_{\text {erg }}$ was zero. Whenever $P_{\text {erg }}$ is zero, $P_{\text {tot }}$ equals $P_{\text {body }}$, and both are negative during this first part of the poling phase at all intensities. We identified a brief period with a clearly negative $P_{\text {tot }}$ for most subjects and conditions, leading us to analyze this issue in more detail: We determined the amount of $W_{\text {tot }}$ and the duration of this period. This period was defined as starting from the onset of poling until $P_{\text {tot }}$ became and remained positive for the remainder of the poling phase (Fig. 3). The duration of energy absorption and the amount of energy absorbed, both

TABLE 1. Physiological and biomechanical characteristics obtained during DP at increasing intensity.

\begin{tabular}{|c|c|c|c|c|}
\hline \multirow[b]{2}{*}{ Variable } & \multicolumn{4}{|c|}{ Intensity } \\
\hline & LOW & MOD & HIGH & MAX \\
\hline Target RPE & 10 & 13 & 16 & 20 \\
\hline Reported RPE & $8.7 \pm 1.7^{*, * *, \star * *}$ & $12.2 \pm 1.5^{\star \star, \star \star * *, \star * \star *}$ & $14.9 \pm 1.3^{*, * * *, * * * *}$ & $19.1 \pm 0.3^{*, * *, * \star * \star}$ \\
\hline$P_{\text {erg }}(\mathrm{W})$ & $116 \pm 16^{\star, \star *, * \star \star}$ & $166 \pm 34^{\star \star, \star * *, \star * \star *}$ & $214 \pm 38^{*, \star \star \star, \star \star \star \star \star}$ & $306 \pm 38^{*, * *, * * * *}$ \\
\hline Cycle rate $(\mathrm{Hz})$ & $0.74 \pm 0.08^{\star \star \star \star \star \star}$ & $0.78 \pm 0.09^{* *, * * *}$ & $0.84 \pm 0.11^{\star, * \star *, * \star \star * *}$ & $0.97 \pm 0.11^{*, * *, * * \star *}$ \\
\hline Poling time (s) & $0.62 \pm 0.06^{\star \star, \star * \star}$ & $0.58 \pm 0.05^{\star *, * \star *}$ & $0.54 \pm 0.04^{\star, * * *, * * * *}$ & $0.49 \pm 0.04^{*, * *, * * * *}$ \\
\hline Poling time $(\%)$ & $45.4 \pm 2.1$ & $44.9 \pm 2.2^{\star \star \star}$ & $44.9 \pm 2.3^{\star * \star}$ & $47.3 \pm 1.8^{\star, \star \star}$ \\
\hline Blood lactate $\left(\mathrm{mmol} \cdot \mathrm{L}^{-1}\right)$ & $1.9 \pm 0.6^{\star, \star \star, \star \star \star}$ & $3.2 \pm 0.8^{\star *, * \star *, \star \star \star * *}$ & $5.7 \pm 0.8^{\star, \star \star \star *, \star \star \star \star}$ & $12.2 \pm 1.8^{\star, \star \star, \star \star \star \star \star}$ \\
\hline $\mathrm{V}_{2}\left(\mathrm{~mL} \cdot \mathrm{kg}^{-1} \cdot \mathrm{min}^{-1}\right)$ & $31.7 \pm 3.9^{\star, \star \star, \star \star \star}$ & $41.2 \pm 6.0^{\star \star, \star \star \star \star, \star \star \star \star \star}$ & $51.3 \pm 7.2^{\star, \star \star *, \star \star \star \star \star}$ & $66.7 \pm 5.1^{\star, \star \star, \star \star \star \star}$ \\
\hline Heart rate (bpm) & $125 \pm 10^{*, * *, * * * *}$ & $147 \pm 10^{* *, * * *, * * * *}$ & $165 \pm 10^{*, * * *, * * * *}$ & $184 \pm 7^{*, * *, * * * *}$ \\
\hline
\end{tabular}

Values are presented as mean $\pm \mathrm{SD}(N=9)$.

${ }^{\star}$ Significantly different $(P<0.05)$ from MOD.

${ }^{*}$ Significantly different $(P<0.05)$ from HIGH.

$* * *$ Significantly different $(P<0.05)$ from MAX.

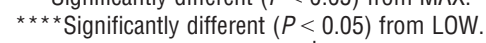

$P_{\text {erg }}$, ergometer poling power; $\dot{V} \mathrm{O}_{2}$, oxygen consumption. 


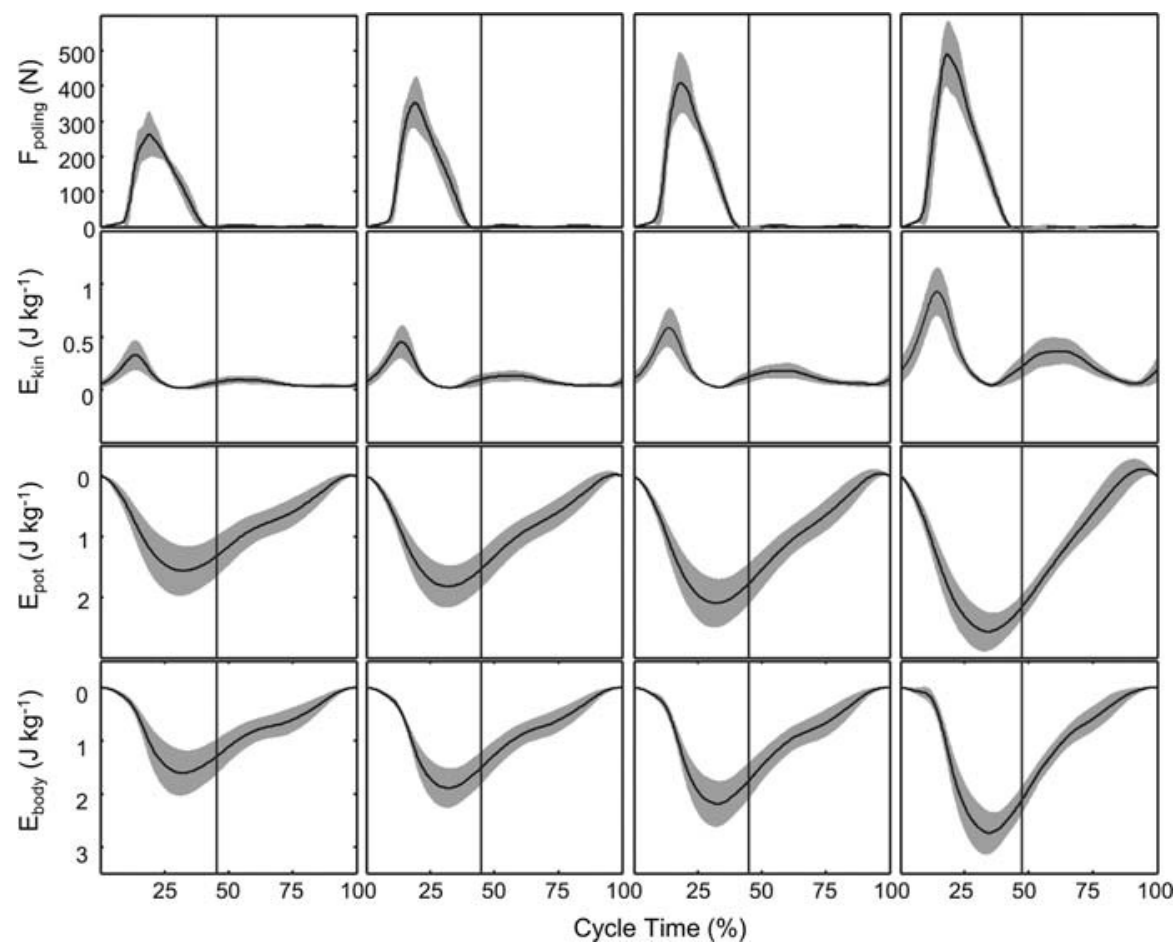

FIGURE 1-Poling force $\left(F_{\text {poling }}\right)$, the sum of segmental kinetic energy $\left(E_{\text {kin }}\right)$ and potential energy $\left(E_{\text {pot }}\right)$, and total mechanical energy $\left(E_{\text {body }}\right)$ plotted as a function of relative cycle time at four different exercise intensities (from left to right: LOW, MOD, HIGH, and MAX) during ergometer DP. $E_{\text {pot }}$ and $E_{\text {body }}$ values are presented relative to energy levels at the start of a cycle $(0 \%)$. The curves show the means for the nine subjects (each subject's trace determined over $\sim 20$ DP cycles under steady-state conditions), and the corresponding SDs are illustrated as shaded areas. The poling phase starts at $0 \%$ of the cycle and ends at the vertical gray lines (the group's mean value).

absolute and relative to $W_{\text {erg }}$, were dependent on intensity $(P<0.01)$ (Table 2). Only at MAX did negative muscletendon work (i.e., $W_{\text {tot }}<0$ ) not differ significantly from zero. For the remainder of the poling phase and throughout the recovery phase, $P_{\text {tot }}$ remained positive (delivering energy). Meanwhile, $P_{\text {body }}$ was negative until approximately $75 \%$ of the poling phase had been completed (i.e., also delivering energy). During the last part of propulsion, $P_{\text {erg }}$ decreased rapidly to zero and $P_{\text {body }}$ became positive, becoming approximately equal to $P_{\text {tot. }}$. During this period, muscle-tendon work increased the mechanical energy of the body. The muscle-tendon work performed was evenly divided between

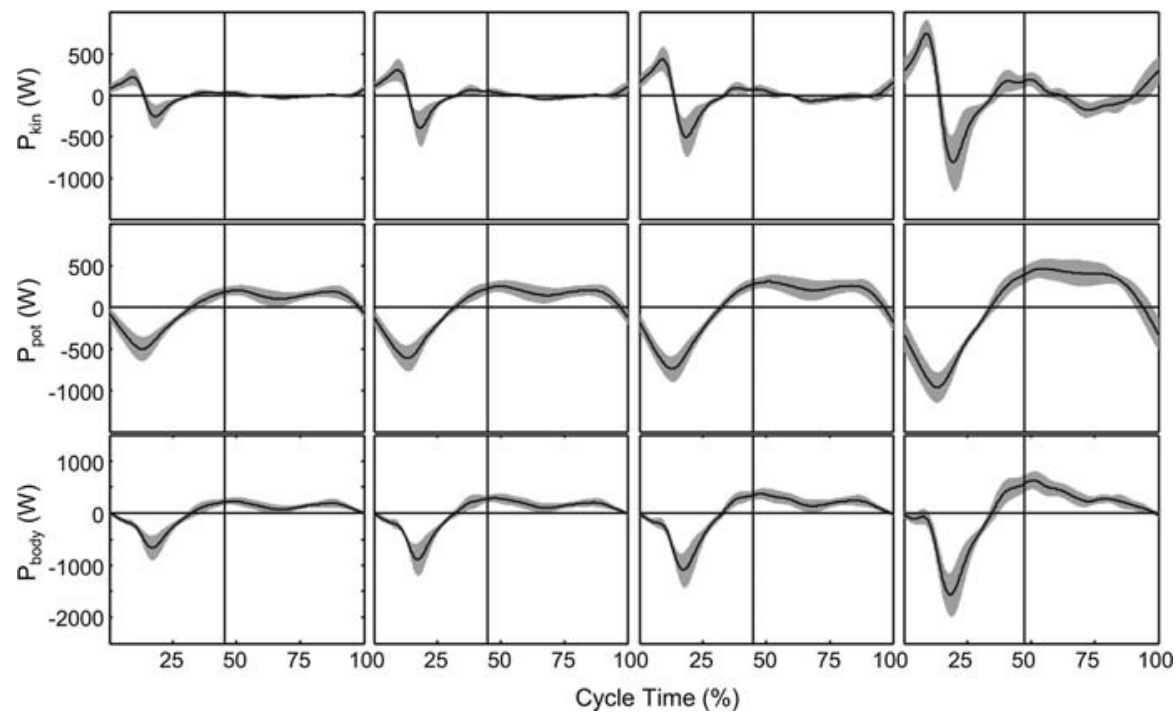

FIGURE 2-The sum of segmental kinetic power $\left(P_{\text {kin }}\right)$, potential power $\left(P_{\text {pot }}\right)$, and total mechanical power $\left(P_{\text {body }}\right)\left(\mathrm{J} \cdot \mathbf{s}^{-1}\right)$ plotted as a function of relative cycle time at four different exercise intensities (from left to right: LOW, MOD, HIGH, and MAX) during ergometer DP. The curves show the means for the nine subjects (each subject's trace determined over $\sim 20$ DP cycles under steady-state conditions), and the corresponding SDs are illustrated as shaded areas. The poling phase starts at $0 \%$ of the cycle and ends at the vertical gray lines (the group's mean value). 


\begin{tabular}{|c|c|c|c|c|}
\hline \multirow[b]{2}{*}{ Variable } & \multicolumn{4}{|c|}{ Intensity } \\
\hline & LOW & MOD & HIGH & MAX \\
\hline$W_{\text {erg }}\left(\mathrm{J} \cdot \mathrm{cycle}^{-1}\right)$ & $157 \pm 21^{*, * \star * \star \star *}$ & $214 \pm 46^{\star \star, \star \star \star *, \star \star \star \star}$ & $257 \pm 47^{*, * \star *, * \star \star \star}$ & $316 \pm 36^{*, \star *, * \star \star *}$ \\
\hline$W_{\text {tot recovery }}\left(\mathrm{J} \cdot\right.$ cycle $\left.^{-1}\right)$ & $104 \pm 26^{\star, * *, * * *}$ & $119 \pm 25^{\star, \star \star, \star \star \star}$ & $140 \pm 26^{\star, * *, * * *}$ & $167 \pm 22^{\star, \star \star, \star \star \star}$ \\
\hline$W_{\text {tot recovery }}(\%)$ & $66 \pm 13^{\star \star \star}$ & $57 \pm 14$ & $55 \pm 8$ & $53 \pm 7^{\star \star \star \star}$ \\
\hline$W_{\text {tot poling }}\left(\mathrm{J} \cdot\right.$ cycle $\left.^{-1}\right)$ & $53 \pm 21^{\star \star * \star \star \star}$ & $95 \pm 49^{\star \star \star}$ & $116 \pm 34^{\star \star \star * \star \star \star \star *}$ & $150 \pm 33^{\star, \star *, \star * \star \star}$ \\
\hline$W_{\text {tot neg poling }}\left(\mathrm{J} \cdot\right.$ cycle $\left.^{-1}\right)$ & $-25 \pm 16^{\star \star \star}$ & $-20 \pm 15^{\star \star \star}$ & $-18 \pm 14^{\star \star \star}$ & $-5 \pm 18^{\star, \star \star, \star \star \star \star}$ \\
\hline$W_{\text {tot neg poling rel }}(\%)$ & $16 \pm 10^{\star \star, * \star *}$ & $10 \pm 9^{\star \star \star}$ & $7 \pm 7^{\star \star \star * \star \star \star \star *}$ & $2 \pm 6^{*, * *, * \star * \star}$ \\
\hline$\triangle E_{\text {kin }}(\mathrm{J})$ & $33 \pm 15^{\star \star, \star \star \star}$ & $47 \pm 16^{\star \star, \star \star \star}$ & $61 \pm 19^{\star, \star \star \star, \star \star \star \star}$ & $98 \pm 26^{*, \star *, * \star \star *}$ \\
\hline$\Delta E_{\mathrm{pot}}(\mathrm{J})$ & $129 \pm 31^{\star, \star \star, \star \star \star}$ & $153 \pm 28^{\star \star, \star \star \star *, \star \star \star \star \star}$ & $176 \pm 33^{\star, \star \star \star, \star \star \star \star \star}$ & $223 \pm 37^{\star, \star *, \star \star \star \star}$ \\
\hline$\triangle E_{\text {body }}(\mathrm{J})$ & $132 \pm 31^{\star, * *, * * *}$ & $156 \pm 28^{* *, * * *, * * * *}$ & $180 \pm 33^{*, * * *, * * * *}$ & $228 \pm 34^{*, * *, * * * *}$ \\
\hline Height CoM max (m) & $1.09 \pm 0.03^{* * *}$ & $1.10 \pm 0.03^{* * *}$ & $1.10 \pm 0.03^{* * *}$ & $1.12 \pm 0.04^{*, * *, * * * *}$ \\
\hline Height CoM min (m) & $0.93 \pm 0.04^{\star, \star *, * \star *}$ & $0.91 \pm 0.04^{* *, * * *, * * * *}$ & $0.88 \pm 0.04^{*, * * *, * * * *}$ & $0.84 \pm 0.04^{*, * *, * \star * *}$ \\
\hline
\end{tabular}

Values are presented as mean $\pm \mathrm{SD}(N=9)$.

* Significantly different $(P<0.05)$ from MOD

** Significantly different $(P<0.05)$ from HIGH.

$* * \star$ Significantly different $(P<0.05)$ from MAX.

$* * * *$ Significantly different $(P<0.05)$ from LOW.

$W_{\text {erg }}$, external ergometer work performed over the cycle; $W_{\text {tot recovery, }}$ muscle-tendon work performed in the recovery phase; $W_{\text {tot }}$ poling, muscle-tendon work performed in the poling

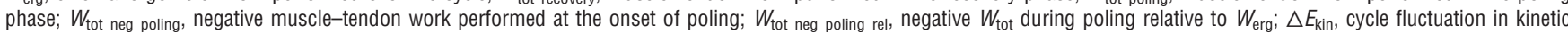
energy; $\triangle E_{\mathrm{pot}}$, cycle fluctuation in potential energy; $\Delta E_{\mathrm{body}}$, cycle fluctuation in total mechanical energy.

poling and recovery $(P>0.1)$, except in the case of LOW, where more $W_{\text {tot }}$ was performed during recovery $(P<0.01)$ (Table 2).

\section{DISCUSSION}

The present investigation of fluctuations in the mechanical energy of the body in relation to fluctuations in external work during DP on an ergometer confirmed our hypothesis: The vertical oscillation of the CoM and related fluctuations in mechanical energy became more pronounced at greater ergometer power outputs. Here, $E_{\text {body }}$ increased due to active leg and upper body muscle-tendon work during the recovery phase and rapidly decreased during the following poling phase when external work was performed. During the poling phase, a "direct" transfer of $E_{\text {body }}$ to external work was therefore assumed to be the main factor contributing to propulsion due to out-of-phase fluctuations of energy between the body's mechanical energy and external work. This is in contrast to other forms of locomotion, such as walking and running $(7,8)$ and diagonal stride XC skiing (17).

In most studies dealing with the exchange of mechanical energy during locomotion (e.g., Refs. $(7,22)$ ), including the present one, no direct evidence of such exchange (i.e., force and its displacement) is provided. Thus, our conclusions in this respect are based partially on a certain rationale. The conclusion that the body's mechanical energy is transferred "directly" to external work is based on out-of-phase fluctuations in body mechanical energy and external work.

In theory, all of the reductions in $E_{\text {body }}$ could be absorbed by the lower extremities in a countermovement-like action whereas, at the same time, all external work is performed by the upper extremities. Although the onset of poling involves energy absorption (Fig. 3), it is unlikely that positive $P_{\text {tot }}$ is a combination of power performed at the upper extremities and power absorbed by the lower extremities. The most likely mechanism is that the lower extremities increase the body's mechanical energy during recovery, which is in turn "reutilized" during propulsion via the upper extremities and poles (ropes in the present study). Because the ergometer setup does not allow any external work to be performed directly by the lower extremities, the abovementioned mechanism is the only way that the lower extremities can contribute to external work. The main physical constraints in DP on skis are similar to the current setup, and no propulsion directly through the skis is possible as long as the skis continuously glide. Thus, the mechanism described

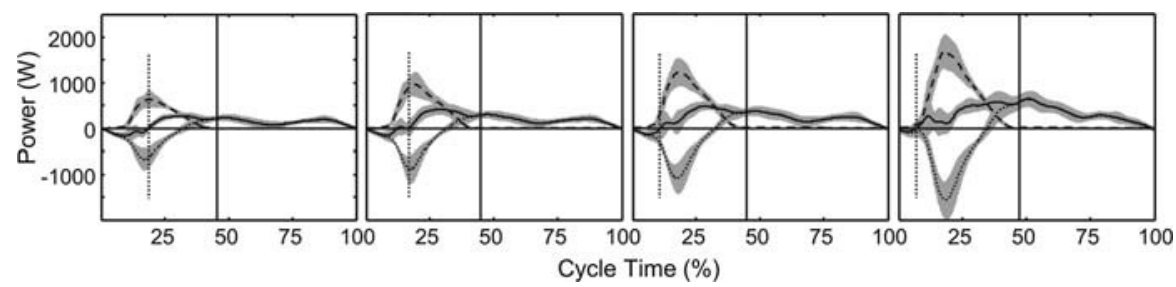

FIGURE 3-External ergometer power $\left(P_{\text {erg }}\right.$; dashed-dotted line), change in the mechanical energy of the body $\left(P_{\text {body }}\right.$; dotted line), and the sum of the two $\left(P_{\text {tot }}\right.$; solid line) plotted as a function of relative cycle time at four different exercise intensities (from left to right: LOW, MOD, HIGH, and MAX) during ergometer DP. The curves show the means for the nine subjects (each subject's trace determined over $\sim 20$ DP cycles under steady-state conditions), and the corresponding SDs are illustrated as shaded areas. The poling phase starts at $0 \%$ of the cycle and ends at the vertical gray lines (the group's mean value). The dashed vertical lines represent the end of the period during which $\boldsymbol{P}_{\text {tot }}$ was negative (see "Methods" for details). 
previously probably applies to DP on skis as well, although future research involving roller skiing and skiing on snow should examine this further.

In the present study, $E_{\mathrm{kin}}$ and $E_{\mathrm{pot}}$ fluctuate out of phase only at the very end of the recovery phase and the very first part of the poling phase, whereas $E_{\text {body }}$ is almost constant and $P_{\text {erg }}$ is close to zero. This indicates that the body is approximately in free fall (i.e., the only significant force acting on the body is gravity). Indeed, the ropes provide no force, but the absence of ground reaction forces needs to be confirmed. Otherwise, fluctuations in $E_{\text {body }}$ during DP are largely out of phase with $P_{\text {erg }}$, indicating a direct transfer of mechanical energy to doing external work (as described in detail previously).

Furthermore, at the end of the recovery phase, fluctuations in $P_{\text {tot }}$ around zero are apparent (Fig. 3), indicating that energy absorption and work generation by muscles-tendons must have occurred. Some energy absorption could be associated with friction; however, in the current setup, with the feet placed statically on the ground and with only a simple set of pulleys between the force sensor and registration of movement, it is unlikely that all negative $P_{\text {tot }}$ can be accounted for by friction. Thus, questions arise on whether a stretchshortening cycle in muscle-tendon systems may have occurred and, if so, whether this was in the upper or lower extremities.

Energy absorption (negative $P_{\text {tot }}$ ) occurred during the very onset of the poling phase when little or no external work was performed. This implies that the reduction in $E_{\text {body }}$ could not have been absorbed by the upper extremities because of a lack of braking forces. Much of the energy absorption, therefore, probably occurred in the lower extremities (i.e., through hip, knee, and ankle flexion). At first sight, it may seem as if the energy absorbed might have been reutilized because the period of energy absorption is followed directly by a period of doing muscle-tendon work. However, this work performed on the ergometer could not have originated from muscles in the lower extremity and thus contradicts such a mechanism. A deeper analysis of dynamics of DP is needed to elucidate this issue.

Nonetheless, considerable active muscle-tendon work must have been performed as well because the amount of energy generated greatly exceeded the amount absorbed (Table 2). Energy absorption at the onset of the poling phase is modest, which is an indication of the main difference between DP and spring-mass styles of locomotion: Instead of storing energy in elastic form during impact, such as during running (e.g., Refs. $(3,13))$, most of the body's mechanical energy during DP is used directly to do external work. The small countermovement-like action that seems to occur at submaximal levels may be related to regulating the amount of work that needs to be delivered from $E_{\text {body }}$ to $W_{\text {erg }}$, rather than representing the typical stretch-shortening mechanism in the classical sense of the term.

Therefore, during the poling phase, two energy sources drive propulsion: direct transfer of the body's mechanical energy to external work and active muscle-tendon work. At the end of the poling phase, muscle-tendon work is converted into $E_{\text {body }}$ rather than $W_{\text {erg }}$, but there is a short overlapping period during which muscle-tendon work is converted into both. During the recovery phase, active muscle-tendon work is performed to restore $E_{\text {body }}$ to its value at the onset of the poling phase.

Although ergometer simulation of DP clearly has limitations with regard to interpretation of actual DP when skiing, an advantage of the present setup was the instantaneous recording of external mechanical work separate from changes in the mechanical energy of the body. In contrast, Pellegrini et al. (22) studied DP roller skiing on a treadmill and concluded, based on the phase relationship between mechanical energy fluctuations, that DP involves inverted pendulum locomotion. Our conclusions contradict those of Pellegrini et al. (22). However, in their analysis (22), horizontal changes in $E_{\text {kin }}$ and a continuous increase in $E_{\text {pot }}$ due to treadmill incline were included in energy traces. In other words, their data on $E_{\text {kin }}$ and $E_{\text {pot }}$ include what in our study would have been defined as external work (i.e., $W_{\text {erg }}$ ). If the increase in $E_{\text {pot }}$ during an entire cycle is subtracted from their $E_{\text {pot }}$ data (i.e., making equal the values at $0 \%$ and $100 \%$ of the cycle) (see Fig. 2 in Pellegrini et al. (22)), a pattern close to that in our study is obtained.

Based on the data presented (22), it is more difficult to account for fluctuations in $E_{\text {kin }}$ in the direction of propulsion, but a similar compatibility with our findings is possible. Thus, the opposite conclusions drawn here and by Pellegrini et al. (22) are not necessarily due to differences between DP on an ergometer and DP on skis. We are critical towards the conclusion drawn by Pellegrini et al. (22) with regard to the overall mechanics of locomotion, particularly because the mechanism that would make the inverted pendulum behavior possible is absent.

Effects of intensity. The difference between the body's mechanical energy utilized during the poling phase and the external work performed was dependent on intensity. At submaximal intensities, the rate of reduction of the body's mechanical energy at the onset of the poling phase exceeded the requirements for external power $\left(P_{\text {tot }}<0\right)$, and energy absorption by muscle-tendon structures probably occurred. The situation changed at maximal intensity-all mechanical energy $\left(E_{\text {body }}\right)$ was utilized directly for propulsion (and, in addition, muscle-tendon work was actively generated very early on).

When considering the entire poling phase, with increasing intensity, the amount of muscle-tendon work and the contribution of the body's mechanical energy increased in about the same proportion (Table 2). The more pronounced fluctuations in $E_{\text {body }}$ with increasing intensity were achieved not primarily by elevating the CoM to a higher position at the end of recovery (which occurred only during MAX) but primarily by lowering the CoM more during the poling phase (Table 2). These findings are in agreement with several studies on the effects of speed (i.e., intensity) on 
adaptation of the DP technique $(15,16,19,21)$. For example, Holmberg et al. (15) found an increased range of motion in the knee and ankle joints during the poling phase at faster DP speeds.

Except for the lowest intensity level, the division of muscle-tendon work performed during the poling and recovery phases remained almost unchanged with increasing intensity: About $50 \%$ of this work was performed during recovery, presumably representing work performed by musclestendons of the lower extremities and trunk. Using a DP ergometer with poles at work rates below (82 W) and comparable to our LOW condition (117 W), Rud et al. (25) found that the work performed by the arms and legs was evenly distributed across the cycle. Increasing ergometer power output $(117 \mathrm{~W})$ was achieved more by the legs than by the arms, as indicated by biomechanical data and oxygen uptake (25).

If we assume that the division between poling and recovery in the present study reflects muscle-tendon work performed by the upper and the lower extremities, respectively, then our results are in disagreement with those of Rud et al. (25) because $W_{\text {tot }}$ during recovery decreased steadily from LOW to MAX (Table 2). Of course, our assumption may not be entirely correct. However, the mechanics analysis by Rud et al. (25) can only be regarded as a rough estimate of the work performed by the legs and is inconclusive with regard to instantaneous transfer of mechanical energy. Concerning the actual muscle-tendon work performed by specific upper-body and lower-body joints in different phases of the movement cycle, future studies can further our understanding by carrying out, for example, inverse dynamics analysis.

The present study confirms previous reports (e.g., Ref. (15)) in the sense that DP involves whole-body movement, and we have demonstrated how the high-hip/high-heel strategy (16) allows utilization of the mechanical energy of the body for propulsion. Apart from elevating the CoM during

\section{REFERENCES}

1. Alexander RM. Energy-saving mechanisms in walking and running. J Exp Biol. 1991;160:55-69.

2. Alexander RM, Vernon A. The mechanics of hopping by kangaroos. J Zool. 1975;177(2):265-303.

3. Biewener AA. Patterns of mechanical energy change in tetrapod gait: pendula, springs and work. J Exp Zool A Comp Exp Biol. 2006;305(11):899-911.

4. Biewener AA, Baudinette R. In vivo muscle force and elastic energy storage during steady-speed hopping of tammar wallabies (Macropus eugenii). J Exp Biol. 1995;198:1829-41.

5. Bobbert MF, Santamaría S. Contribution of the forelimbs and hindlimbs of the horse to mechanical energy changes in jumping. J Exp Biol. 2004;208:249-60.

6. Cavagna GA. Force platforms as ergometers. J Appl Physiol. 1985;39(1):174-9.

7. Cavagna GA, Heglund NC, Taylor CR. Mechanical work in terrestrial locomotion: two basic mechanisms for minimizing energy expenditure. Am J Physiol. 1977;233(5):243-61.

8. Cavagna GA, Saibene FP, Margaria R. Mechanical work in running. J Appl Physiol. 1964;19(2):249-56.

9. Cavagna GA, Thys H, Zamboni A. The sources of external work in level walking and running. J Physiol. 1976;262:639-57. recovery, subtle timing of body motion for effective mechanical energy transfer appears to be of considerable importance. However, in future studies, ergometer DP should be compared carefully to DP on snow before detailed recommendations concerning the DP technique can be made.

\section{CONCLUSIONS}

The present study shows that, during DP locomotion, skiers generate large fluctuations in $E_{\text {body }}$. By increasing the body's mechanical energy during the recovery phase, this energy can subsequently be transferred directly into doing external work during the poling phase. The out-of-phase fluctuations between $E_{\text {body }}$ and external power (i.e., power applied to the ergometer) indicate that DP does not resemble spring-mass behavior. Instead, the work performed by the lower extremities during the recovery phase is "stored" as the body's mechanical energy, to be reutilized during the poling phase. At submaximal intensities, a small amount of the energy released is probably "recovered" during a bouncing countermovement-like action. This may be a result of having an abundance of mechanical energy available, or the bounce may potentiate muscle force production.

The authors would like to express their gratitude to the participating athletes and their coaches for their cooperation and enthusiasm during the testing sessions. The authors thank Xiang Chun Tan (Department of Neuroscience, Norwegian University of Science and Technology, Trondheim, Norway) for all her help in data collection. No funding was received for the present study.

None of the authors have any relevant conflicts of interest to declare.

All authors assisted in the writing of the manuscript and were involved in the study design and/or data collection, analysis, and interpretation.

The results of the present study do not constitute endorsement by the American College of Sports Medicine.

10. de Leva P. Adjustments to Zatsiorsky-Seluyanov's segment inertia parameters. J Biomech. 1996;29(9):1223-30.

11. Ettema G. Mechanical efficiency and efficiency of storage and release of series elastic energy in skeletal muscle during stretch-shorten cycles. J Exp Biol. 1996;199:1983-97.

12. Ettema G. Muscle efficiency: the controversial role of elasticity and mechanical energy conversion in stretch-shortening cycles. Eur J Appl Physiol. 2001;85:457-65.

13. Farris DJ, Sawicki GS. The mechanics and energetics of human walking and running: a joint level perspective. $J R$ Soc Interface. 2012;9:110-8.

14. Halonen J, Ohtonen $\mathrm{O}$, Lemmettylä $\mathrm{T}$, et al. Biomechanics of double poling when skiing on snow and using an ergometer. In: Müller E, Kröll J, Lindinger S, Pfusterschmied J, Stöggl T, editors. Science and Skiing VI. Germany: Meyer \& Meyer Sport (UK) Ltd; 2015. pp. 387-95.

15. Holmberg HC, Lindinger S, Stöggl T, Björklund G, Müller E. Contribution of the legs to double-poling performance in elite cross-country skiers. Med Sci Sports Exerc. 2006;38(10):1853-60.

16. Holmberg HC, Lindinger S, Stöggl T, Eitzlmair E, Müller E. Biomechanical analysis of double poling in elite cross-country skiers. Med Sci Sports Exerc. 2005;37(5):807-18. 
17. Kehler AL, Hajkova E, Holmberg HC, Kram R. Forces and mechanical energy fluctuations during diagonal stride roller skiing; running on wheels? J Exp Biol. 2014;217:3779-85.

18. Lindinger SJ, Holmberg HC. How do elite cross-country skiers adapt to different double poling frequencies at low to high speeds? Eur J Appl Physiol. 2011;111(6):1103-19.

19. Lindinger SJ, Stöggl T, Müller E, Holmberg HC. Control of speed during the double poling technique performed by elite crosscountry skiers. Med Sci Sports Exerc. 2009;41(1):210-20.

20. Minetti AE. Passive tools for enhancing muscle-driven motion and locomotion. J Exp Biol. 2004;207(8):1265-72.

21. Nilsson J, Tinmark F, Halvorsen K, Arndt A. Kinematic, kinetic and electromyographic adaptation to speed and resistance in double poling cross country skiing. Eur J Appl Physiol. 2013;113(6): 1385-94.

22. Pellegrini B, Zoppirolli C, Bortolan L, Zamparo P, Schena F. Gait models and mechanical energy in three cross-country skiing techniques. J Exp Biol. 2014;217:3910-8.
23. Pezzack JC, Norman RW, Winter DA. An assessment of derivative determining techniques used for motion analysis. $J$ Biomech. 1977;10:377-87.

24. Pfau T, Witte TH, Wilson AM. Centre of mass movement and mechanical energy fluctuation during gallop locomotion in the Thoroughbred racehorse. J Exp Biol. 2006;209:3742-57.

25. Rud B, Secher NH, Nilsson J, Smith G, Hallén J. Metabolic and mechanical involvement of arms and legs in simulated double poling skiing. Scand J Med Sci Sports. 2014;24:913-9.

26. Seiler S, Tønnessen E. Intervals, thresholds, and long slow distance: the role of intensity and duration in endurance training. Sportscience. 2009;13:32-53.

27. van Ingen Schenau GJ, Bobbert MF, de Haan A. Does elastic energy enhance work and efficiency in the stretch-shortening cycle? J Appl Biomech. 1997;13:389-415.

28. Winter DA. A new definition of mechanical work done in human movement. J Appl Physiol. 1979;46(1):79-83.

29. Winter DA. Biomechanics and Motor Control of Human Movement. New York (NY): John Wiley \& Sons; 1990: p. 325. 